\title{
Metacommunicative devices in spoken discourse as part of processing distributed cognitive tasks
}

\author{
Ilya Utekhin, Tatiana Chernigovskaya \\ Department of General Linguistics, St.Petersburg State University, Russia \\ https://doi.org/10.36505/ExLing-2011/04/0036/000205
}

\begin{abstract}
Types of utterances that belong to meta-levels of activity have been singled out in spoken task-focused dialogues between subjects accomplishing a matching task with no visual contact. The types include activity management and planning, global and local (e.g. signals of activity phase); explicit evaluation of the state of joint project, of one's own state or partner's state; retrospective references and accounts; metacommunicative utterances for conversation management; backchannel response: "continuers"; collaborative completion, echoic quotation; repair (self-repair, otherrepair); meta-linguistic utterances; meta-communicative utterances having to do with rapport, etiquette, joking, etc. Differences between normal and schizophrenic subjects have been shown in meta-communicative activity supposed to be linked to the management of distributed cognition and creation of shared representations of reality.
\end{abstract}

Key words: conversation, interaction, matching task, metacommunication

\section{Matching task: a joint activity}

The project was aimed at exploring cognitive tools, linguistic techniques and discursive devices spontaneously formed by subjects who participated in an experimental study of interaction in dyads: two subjects accomplished experimental task with an opaque screen preventing them from seeing each other's face and workspace. One of the subjects (Director) explained her partner (Matcher) how to assemble Lego blocks into a model to match the prototype or, in another series, how to arrange photographs of cloudy sky on the table, in order to match the source layout visually available to Director only.

Lack of visual access to the partner's field of operation emphasizes the role of coordination, or alignment, of perspectives between the partners. Participants' construal of each other's view can only rely on spoken discourse, without visual feedback concerning partner's actions and understanding. Accordingly, mechanisms of reference, anaphora, conceptual choice, and deictic anchorage of utterances function in somewhat special ways under these conditions compared to face-toface conversation in a shared space where pointing gestures and visual monitoring of each other's activity are available.

Matching task paradigm has already been used for the study of communicative interaction since Piagetian studies of referential communication dating back to $1920 \mathrm{~s}$, cf. more recent

ExLing 2011: Proceedings of 4th Tutorial and Research Workshop on Experimental Linguistics, 25-27 May, Paris, France 
developments in Wilkes-Gibbs, Clark (1992); Metzing, Brennan (2003); Clark, Krych (2004), and also a concise review in Schober (2006). In our version, in both Lego and photograph tasks, each partner acted as Director and Matcher consecutively. Pairs of subjects comprised normal adults, young children and adult schizophrenic patients, in homogeneous as well as mixed pairs composed of normal adults interacting with schizophrenic adults, and normal adults interacting with children, totalling 200 episodes of interaction, videotaped and transcribed to form a multimedia corpus. The rationale behind involving schizophrenic patients is the claim that schizophrenic patients suffer from a specific impairment of the ability to deduce the mental state of communicative partner, though their cognitive abilities remain unaffected, see Brune (2005) for review of literature. We looked for the features of communicative interaction typical for schizophrenics' less efficient performance in experimental tasks compared to normal controls. We also studied communicative strategies used by more cognitively and communicatively efficient partner in mixed pairs to compensate their partner's deficiency.

Since matching task involves partners' partial access to reality and critically depends on cooperation, it cannot be reduced to partners' individual actions. We consider this to be a case of distributed cognition, as conceived by E.Hutchins (1995). It is essentially public because the control of activity is performed by means of spoken discourse.

\section{Dialogue and the management of activity: meta-level phenomena}

During the interaction, partners' contributions to dialogue organize the activity of task accomplishment and also work for internal organization of the dialog itself, providing for turn-taking, display of understanding, monitoring partner's understanding, etc. Dialogue work is particularly evident in the phenomenon of repair (including self-repair, other-repair, and repair initiation). As conceptualised within conversation analytical paradigm, repair demonstrates participants' concern with mutually certified understanding: the absence of repair after the next turn means by default that partner's understanding of the first turn was accepted by the speaker, see, e.g., Schegloff (1992) and elaboration of this logic in the notions of presentation phase and acceptance phase by H.Clark (1996). Self-repair and related disfluencies can be psycholinguistically explained by internal monitoring of one's own speech production; for classification of self-repair in a corpus of task-oriented dialogues, see studies by Levelt, starting from Levelt (1983).

Studies by Clark and associates have shown that what is going on in matching task dialogues mostly fits within identification and localization phases performed consecutively for all the elements. We have studied 
optional verification sequences, as well as some other types of contributions like in this example (quoted in translation from Russian):

M[atcher]: we should win

$\mathrm{D}$ [irector]: wow!

$>$ M: let's start with the simple ones.

$\mathrm{D}$ : if they were labeled "this is a simple one"... (LAUGHS)

M: those which have blue spots

D: you've got four pics with blue spots

>M: ok. well, here I provisionally set apart some four

D: four

In the marked lines, utterances have to do with general management of activity, whereas local management can be exemplified with signalling or requesting about readiness to go to a next step. Other meta-level activities considered in this study include: explicit evaluation of the state of joint project, of one's own state or partner's state; retrospective references and accounts; meta-communicative utterances for conversation management; backchannel response: "continuers"; display utterances: collaborative completion, echoic quotation; meta-linguistic utterances; metacommunicative utterances having to do with rapport, etiquette, joking. If present in a participant's speech, these types of contributions to dialogue can be regarded as features of interactivity, involvement, and concern with intersubjectivity.

\section{Results: norms and pathology}

Normal participants compared against schizophrenic patients show significantly more relevant utterances . This correlates with greater flexibility in choosing strategies of joint activity. Thus, unlike in schizophrenia, the normal Matchers readily switch from matching to describing, when Director has difficulties in explaining. Some types of utterances better correspond to Director's or Matcher's role, cf., for instance, general activity management seems to correspond to Director's endeavour.

Table 1. General activity management utterances, present in episodes, per cent.

\begin{tabular}{|c|c|c|c|}
\hline \hline \multicolumn{2}{|c|}{ Pathology } & \multicolumn{2}{c|}{ Norms } \\
\hline Directors & Matchers & Directors & Matchers \\
\hline $44 \%$ & $22 \%$ & $86 \%$ & $71 \%$ \\
\hline
\end{tabular}


Utterances that display understanding and acceptance, particularly, echoic repetition of partner's word(s) and collaborative completion of partner's utterances, also differ in norms and pathology, leading to a conclusion that for some reason cognitively non-impaired schizophrenic patients with fluent speech show less interest in actively updating a common ground in dialogue.

Table 2. Display utterances (collaborative completion, echoic response), average per episode.

\begin{tabular}{|c|c|c|c|}
\hline \hline \multicolumn{2}{|c|}{ Pathology } & \multicolumn{2}{c|}{ Norms } \\
\hline Directors & Matchers & Directors & Matchers \\
\hline 1.0 & 1.6 & 2.5 & 4.5 \\
\hline \hline
\end{tabular}

Pathology demonstrates a deficit of explicit planning and coordination of joint activities, reduced or lacking verifications, and a lack of metalinguistic utterances. Explicit metacommunicative utterances, although differently across their types, are also less numerous in pathology. In mixed pairs, metacommunicative utterances by the normal partner seem to be a means to enhance the dyad's efficiency.

\section{Acknowledgements}

Grants from the Russian Ministry of Education and Science (\# 16.740.11.0113, $02.740 .11 .0369)$

\section{References}

Brune, M. 2005. "Theory of mind" in schizophrenia: A review of literature. Schizophrenia Bulletin 31, 1, 21-42.

Clark, H.H. 1996. Using Language. Cambridge: Cambridge University Press.

Clark, H.H., Krych, M.A. 2004. Speaking while monitoring addressees for understanding. Journal of Memory and Language 50, 62-81.

Hutchins, E. 1995. Cognition in the Wild. Cambridge (MA): MIT

Levelt, W. 1983. Monitoring and self-repair in speech. Cognition 14, 41-104.

Metzing, C., Brennan, S.E. 2003. When conceptual pacts are broken: partnerspecific effects in the comprehension of referring expressions. Journal of Memory and Language 49, 201-213.

Schegloff, E. 1992. Repair after next turn: The last structurally provided defence of intersubjectivity in conversation. Americal Journal of Sociology 97, 5, 1295-1345.

Schober, M.F. 2006. Dialogue and Interaction. In Brown K., (Editor-in-Chief) Encyclopedia of Language \& Linguistics, Second Edition, volume 3, 564-571. Oxford: Elsevier.

Wilkes-Gibbs, D., Clark H.H. 1992. Coordinating beliefs in conversation. Journal of Memory and Language 31, 183-194. 